\title{
THE MASS DISTRIBUTION OF SECONDARIES TO SOLAR-TYPE STARS
}

\author{
Helmut A. Abt ${ }^{1}$ and Daryl W. Willmarth ${ }^{1}$ \\ RESUMEN
}

Se muestra que dos estudios previos sobre la distribución de las masas de las secundarias de las binarias espectroscópicas (Abt \& Levy 1976; Duquennoy \& Mayor 1991) concuerdan bien si ambos se grafican con la misma escala en las abscisas. Un nuevo estudio de 271 estrellas de la secuencia principal más tardías que F6 $\mathrm{V}$, realizado con una precisión en las velocidades radiales de $\pm 0.10 \mathrm{~km} \mathrm{~s}^{-1}$ nos permitió obtener elementos orbitales para 10 sistemas nuevos, además de los 59 ya publicados. La función de masa para las secundarias que resulta es casi plana, y muestra que $2.2 \pm 1.5 \%$ de las primarias tienen compañeras de baja masa $(0.01-0.10$ $\left.M_{\odot}\right)$. En contraste, la función de masa de las compañeras de las binarias visuales con separaciones $>500 \mathrm{AU}$ se ajusta a la función de van Rhijn, como lo demostraron Abt y Levy con anterioridad.

\section{ABSTRACT}

Two previous studies of the secondary mass function in spectroscopic binaries by Abt \& Levy (1976) and by Duquennoy \& Mayor (1991) are shown to be in good agreement if they are both plotted with the same abscissa scale. A new study of 271 main-sequence stars later than F6 V made with a radial-velocity accuracy of $\pm 0.10 \mathrm{~km} \mathrm{~s}^{-1}$ yielded 10 new sets of orbital elements in addition to the 59 published ones. The resulting secondary mass function is nearly flat and shows that $2.2 \pm 1.5 \%$ of the primaries have low-mass $(0.01-0.10$ $\left.M_{\odot}\right)$ companions. In contrast, the secondary mass function for visual binaries with separations $>500 \mathrm{AU}$ fits a van Rhijn function, as was shown previously by Abt and Levy.

\section{Key Words: BINARIES: SPECTROSCOPIC - STARS: LUMINOSITY, MASS FUNCTION}

\section{INTRODUCTION}

The goals of this project are to determine the mass function of secondaries in solar-type binaries, to learn whether that differs for closely-spaced (spectroscopic) and widely-spaced (visual) binaries, and to determine the frequency of brown dwarfs.

In the past there were two major studies of the frequencies of the secondaries of solar-type stars. The one by Abt \& Levy (1976) was based on photographic spectra and had an accuracy of $\pm 1.4 \mathrm{~km} \mathrm{~s}^{-1}$. They found that the numbers of secondaries in visual doubles fit the van Rhijn luminosity function but those in spectroscopic binaries decreased with decreasing mass.

The study by Duquennoy \& Mayor (1991) was done with CORAVEL and had an accuracy of \pm 0.31 $\mathrm{km} \mathrm{s}^{-1}$. They found an increasing frequency of secondaries with decreasing mass. Because of their better accuracy, their conclusion seemed preferable to the Abt \& Levy study. What was not noticed was that the Abt \& Levy abscissa was on a logarithmic scale while that of Duquennoy \& Mayor was on a linear scale. If both are plotted on the same scale (Fig. 1) the agreement is good.

Neither study had the accuracy to explore well

\footnotetext{
${ }^{1}$ Kitt Peak National Observatory, Tucson, AZ, USA.
}

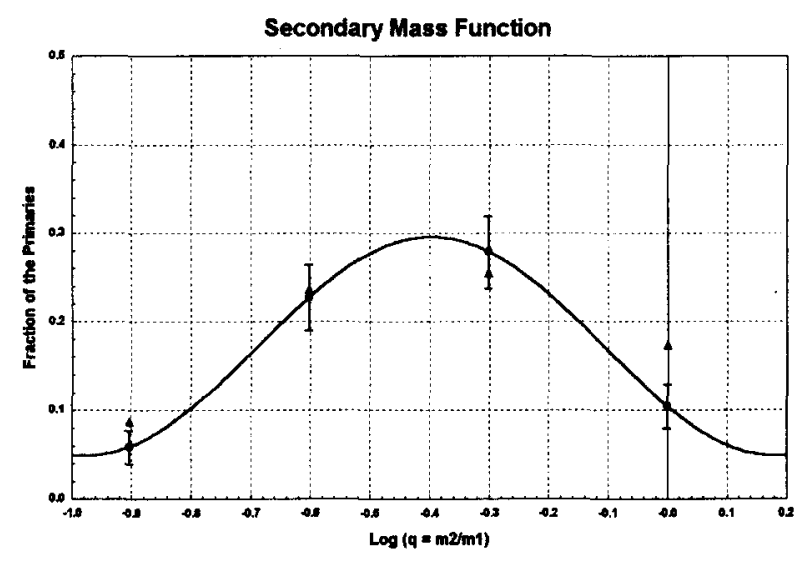

Fig. 1. Comparison of the secondary mass functions by Duquennoy \& Mayor (1991), shown by dots and error bars, and by Abt \& Levy (1976), shown by triangles. The abscissas are on a logarithmic scale relative to the primary masses. The ordinates are relative to the total sample of primaries. These are for all orbital periods. The agreement between the two studies is within the estimated errors.

the brown-dwarf region. Duquennoy \& Mayor's estimate was that " $(8 \pm 6) \%$ of the total sample of primaries could have a VLMC [very low mass companion] in the mass range of $0.01-0.10 M_{\odot} . " \mathrm{Abt} \&$ 
Levy suggested about $7 \%$. Thus it was suspected more than a decade ago the brown dwarfs are probably found in spectroscopic binaries.

Recently it has been found that, among single stars, brown dwarfs are frequent (Gizis et al. 2001). Reid et al. (1999) concluded that "brown dwarfs are twice as common as main-sequence stars." However, as secondaries in binary systems, Halbwachs et al. (2000) found from an Hipparcos study of 11 spectroscopic binaries and from statistical arguments that brown dwarfs are rare in binaries. Using very precise radial velocities, Campbell, Walker and Yang (1988) found no brown dwarfs in 12 late-type spectroscopic binaries. Marcy and his colleagues have found more than 100 binary companions with $M_{2} \sin i$ values less than those of M-type dwarfs. They (Marcy \& Butler 2000) concluded that because they found less than $0.5 \%$ of their primaries to be brown dwarfs, there is a "brown dwarf desert" so that small values of $M_{2} \sin i$ must imply small values of $M_{2}$.

A possible reason can be given for high browndwarf frequency among field stars and a low browndwarf frequency in binaries if binaries are usually formed in three-body interactions in clusters. Aarseth \& Hills (1972), and several talks during this conference, have suggested that in such encounters the lowest-mass components, e.g. the brown dwarfs, are ejected from the more massive pairs and sometimes are ejected from the cluster. However, the brown-dwarf frequency must still be determined from an objective sample, not from one that avoids known visual or spectroscopic binaries or stars with large velocity variations. Also, for an expected frequency between 0.5 and $8 \%$, the sample studied should have several hundred stars, not one or two dozen. For instance, if the frequency turned out to be $5 \%$ and the sample was 200 stars, the accuracy would be $1.6 \%$.

\section{NEW STUDY}

We made a study of 287 stars, using the Kitt Peak $0.9 \mathrm{~m}$ coudé-feed telescope, an optical fiber in place of a slit, and observations within two hours of the meridian. The latter two conditions were to minimize atmospheric dispersion effects. We also had two fibers for thorium-argon comparison spectra. When we compare our observations of 105 "constantvelocity" stars by Nidever et al. (2002), we derive a mean accuracy of $\pm 0.10 \mathrm{~km} \mathrm{~s}^{-1}$. This accuracy is insufficient to discover planets, but it should identify most brown dwarfs.

We observed all the F7 V to $\mathrm{K} 7 \mathrm{~V}$ stars in the Bright Star Catalogue. That sample, based on the
Harvard Revised Photometry, was intended in 1908 to be complete to $\mathrm{V}=6.5 \mathrm{mag}$, but they missed some brighter stars and included many fainter ones, as faint as 7.6 mag. Our sample is not magnitude or distance limited, but because the selection of stars was made in 1908 before much information was available about spectroscopic duplicity, it constitutes a random sample. In fact, the mean apparent magnitudes of $25 \mathrm{SB} 1 \mathrm{~s}(5.63 \pm 0.12 \mathrm{mag})$ and $28 \mathrm{SB} 2 \mathrm{~s}$ $(5.68 \pm 0.13 \mathrm{mag})$ are not brighter than those of 28 constant-velocity stars $(5.44 \pm 0.18 \mathrm{mag})$.

We obtained 4261 measures or an average of 16 per star. The 16 stars classified as luminosity class $\mathrm{V}$ but having Hipparcos parallaxes indicating that they were class III were eliminated, leaving 271 stars.

At the time of this conference we had not analyzed all our velocities, but that has now been done. We used published orbital data for $26 \mathrm{SB} 1 \mathrm{~s}$ and 33 SB2s. We obtained orbital elements for 10 additional SB1s, giving a frequency of spectroscopic binaries of $25 \%$. However there are 27 additional stars in the sample for which orbital elements have not yet been derived. Those either have double lines, velocity dispersions of 0.3 to $5.4 \mathrm{~km} \mathrm{~s}^{-1}$, or evidence for periods much longer than our 1.8 years of observing (plus less accurate data from a similar period in 1987-1989). The possible total of 96 spectroscopic binaries suggests a frequency as high as $35 \%$.

The masses of the secondaries were determined directly for SB2s by assuming that the primaries fit the main-sequence mass-luminosity relation. Those yield secondary masses between 0.6 and $1.3 M_{\odot}$. For the SB1s we assumed a random orientation of orbital axes and identified various derived values of $M_{2} \sin i$ against those expected by probability. To allow for incompleteness we assumed that we were generally unable to resolve SB2s with $K_{1}+K_{2}<22 \mathrm{~km} \mathrm{~s}^{-1}$ or SB1s with $K<3 \mathrm{~km} \mathrm{~s}^{-1}$ (although with only 16 observations per star it is sometimes possible to find orbital elements for smaller amplitudes). Those allowances for undetected companions added 10.1 stars to the total. Those incompleteness calculations were obviously too modest because there are 27 stars with unknown orbital elements, as indicated above. The secondary mass function is shown in Figure 2. Of course there is an upper limit of about $M_{2}=1.3 M_{\odot}$, the mass of the earliest primaries.

We see that 6.0 , or $2.2 \pm 1.5 \%$, of the stars have $M_{2}$ in the range $0.01-0.10 M_{\odot}$. That is based on four stars (HR 145, 3396, 4375, and 5346) with mass functions between $0.04 \times 10^{-6}$ and $52 \times 10^{-6}$. The incompleteness calculations add 2.0 more stars in that range of masses. Thus we find that the fre- 


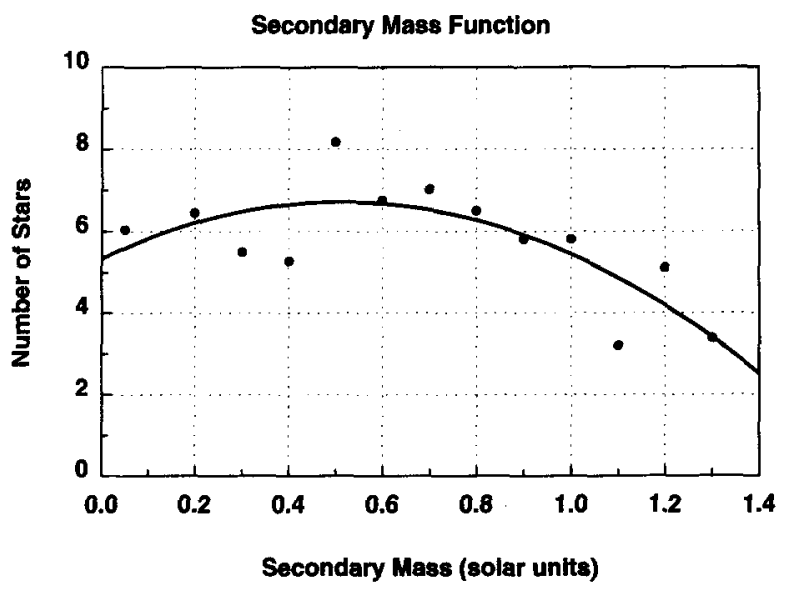

Fig. 2. The secondary mass function from the current study based on 67 spectroscopic binaries plus estimated allowances (10.1 stars) for incompleteness. Relative to a total sample of 271 , the fraction of low-mass companions $\left(0.01-0.10 M_{\odot}\right)$ is $2.2 \%$.

quency of brown dwarfs is significant.

\section{VISUAL BINARIES}

In the on-line Washington Double-Star Catalog we discovered 62 companions (of the 271 primaries) with orbital elements and 45 common-proper-motion companions. Of those 62,24 were already counted as SBs, so we find $38+45=83$ visual binaries for a total binary frequency of at least $56 \%$. Allowance for SBs without orbital elements would raise that to $66 \%$. Therefore more than half the primaries have companions of some kind.

One problem with cataloged data on visual multiples is that visual observers have learned that most stars fainter than 12th mag. and more than $20^{\prime \prime}$ from bright stars prove to be optical companions. Therefore there is an observational bias in the literature against finding secondaries less than $0.4 M_{\odot}$. The secondary mass function for visual pairs more than $500 \mathrm{AU}$ apart is shown in Figure 3. The curve is the van Rhijn function for nearby stars. We see that the fit is within the errors, which are taken as the square roots of the numbers. A similar conclusion was obtained by Abt and Levy.

That function is different from the nearly flat distribution shown in Figure 2 for spectroscopic binaries. However, there is a natural explanation for that difference. Consider the numerical simulations of Aarseth \& Hills (1972) for the formation of bodies by three-body interactions. In the first generations the massive stars acquire whatever companions are most frequently found around them, namely lowmass companions. During further formation and dis-

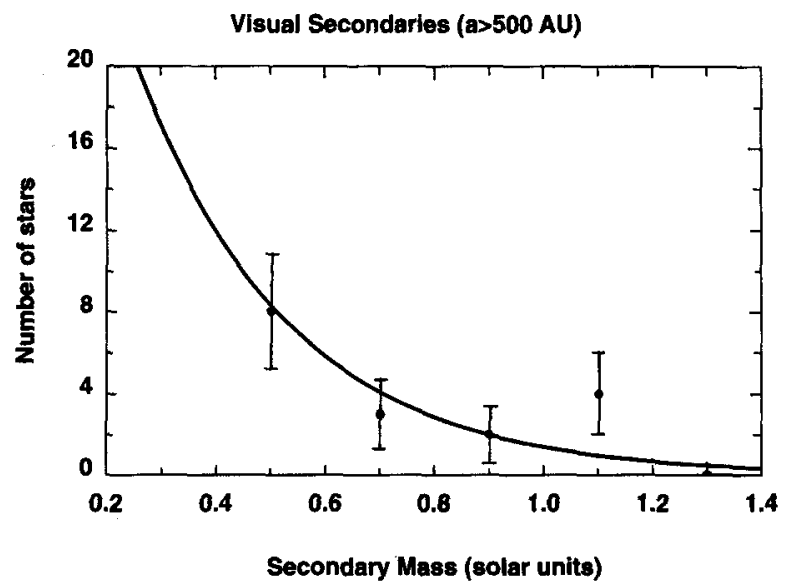

Fig. 3. The secondary mass function for 17 visual binaries with separations a $>500 \mathrm{AU}$. The curve is the van Rhijn function for nearby stars. The agreement indicates that widely spaced binaries fit the van Rhijn function while closely spaced binaries have a flat distribution.

ruption of pairs, the massive stars gradually acquire massive companions and eject the low-mass stars. The visual binaries represent the initial loose pairs formed while the spectroscopic binaries are the tight ones produced after many pairs are formed and disrupted.

The observing for this project was funded by the Research Corporation.

Note added in proof. Regarding the manuscript of a journal article on this material, the referee was not convinced that the sample used was an objective one. He or she showed that SB2s were over-represented. Although that would affect primarily the large-mass end of the mass function in Figure 2, it was decided to define the sample in a different way. The new sample is all the stars within $25 \mathrm{pc}$ as measured with Hipparcos parallaxes and between declination limits $-30^{\circ}$ and $+75^{\circ}$. After additional observing is done, that manuscript will be revised.

\section{REFERENCES}

Aarseth, S., \& Hills, J. G. 1972, A\&A, 21, 255

Abt, H. A., \& Levy, S. G. 1976, ApJS, 30, 273

Campbell, B., Walker, G. A. H., \& Yang, S. 1988, ApJ, 331,902

Duquennoy, A., \& Mayor, M. 1991, A\&A, 248, 485

Gizis, J. E., Kirkpatrick, J. D., Burgasser, A., Reid, I. N., Monet, D. G., Liebert, J., \& Wilson, J. C. 2001, ApJ, 551, L63

Halbwachs, J.-L., Arenou, F., Mayor, M., Udry, S., \& Queloz, D. 2000, A\&A, 355, 581

Marcy, G. W., \& Butler, R. P., PASP, 112, 137

Nidever, D. L., Marcy, G. W., Butler, R. P., Fischer, D. A., \& Vogt, S. 2002, ApJS, 141, 503 
Reid, I. N., Kirkpatrick, J. D., Liebert, J., Burrows, A., Gizis, J. E., Burgasser, A., Dahn, C. C., Monet, D.,
Cutri, R., Beichman, C. A., \& Skrutskie, M. 1999, ApJ, 521, 613

\section{DISCUSSION}

Sterzik - Could you comment about the existence of the "brown dwarf desert" found in precision radial velocity surveys? (Halbwachs et al. 2002, A\&A).

$A b t$ - Observations of single stars show no brown-dwarf desert, so it is important to learn whether there is a deficiency of brown dwarfs in spectroscopic binaries. This is an important question that should be studied by more than one group.

Halbwachs to the question of Sterzik - About the existence of a brown-dwarf desert for short periods: the Coralie sample is a volume-limited sample of 1600 stars, including double systems with companions having masses from the stellar down to the planet domain. There is a brown-dwarf desert for spectroscopic binaries. The same is observed in the Delfosse et al.(1998) M-dwarf Elodie survey.

Upgren - Can you clarify whether relatively massive stars pick up more massive companions at a steady rate, while low-mass stars get captured at a diminishing rate, thus raising the average masses of the companions? Or does the capture rate of higher-mass companions actually increase?

$A b t$ - In the Aarseth-Hills simulations a star picks up one companion at a time. The initial pairs are often disrupted, so the primary loses one star and picks up a different one.

Mardling - By what process do massive stars pick up low-mass stars?

$A b t$ - The "captures" occur in 3-body interactions.

Zinnecker - Is the faint end of the van Rhijn luminosity function really equivalent to the low-mass Salpeter IMF? Your old (1976) result that the secondary mass distribution in low-mass visual binaries follows the van Rhijn luminosity function (or equivalently the Salpeter IMF), reinforced and confirmed by your new measurements that you described today, may not be consistent with a random pairing of low-mass stars drawn from a Salpeter IMF, because: (1) The Salpeter IMF is believed to be too steep at the low-mass end compared with the best current field star IMF determination (cf. Kroupa 2002, Science, 295, 82). (2) Random pairing from a Salpeter IMF does not give a Salpeter (or van Rhijn) function for the secondary masses (cf. Malkov \& Zinnecker 2001, MNRAS, 321, 149).

$A b t$ - I used the distribution function for single stars given in Cox's "Astrophysical Quantities". You are an expert on which values are the best ones. The function is not well known below a peak at about $M_{v}=$ 15 Mag. However, Reid and others find that among single stars brown dwarfs are very frequent.

Helmut A. Abt and Daryl W. Willmarth: Kitt Peak National Observatory, PO Box 26732, Tucson, AAZ 85726-6732, USA (abt@noao.edu). 